\title{
Palliative radiotherapy for thoracic spine metastases: Dosimetric advantage of three-dimensional conformal plans
}

\author{
SEUNG-GU YEO

\begin{abstract}
Department of Radiation Oncology, Soonchunhyang University College of Medicine, Soonchunhyang University Hospital, Cheonan 330-721, Republic of Korea
\end{abstract}

Received August 1, 2014; Accepted March 27, 2015

DOI: $10.3892 / \mathrm{ol} .2015 .3205$

\begin{abstract}
The aim of the present study was to investigate the dosimetric advantages of three-dimensional conformal radiation therapy (3DCRT) for thoracic spine metastases and compare it with conventional two-dimensional (2D) plans. Radiation therapy (RT) planning data of 10 patients with mid-to-low thoracic spine metastases were analyzed. Computed tomography simulation was performed and the planning target volume (PTV), heart, esophagus, lung and spinal cord were contoured. The 3DCRT plan comprised one posteroanterior (PA) field and two posterior oblique fields. The 2D plans used a single PA field or opposed anteroposterior (AP)/PA fields. The prescription dose of radiation was 30 Gy in 10 fractions. All comparisons of the maximum or mean doses to the organs at risk or the PTV, between two of the three RT plans, demonstrated statistically significant differences $(\mathrm{P}<0.05)$, with the exception of the mean esophageal doses between the single PA vs. AP/PA $(\mathrm{P}=0.285)$

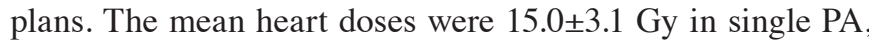
$17.3 \pm 4.3 \mathrm{~Gy}$ in AP/PA and $8.5 \pm 1.7 \mathrm{~Gy}$ using 3DCRT. The median reduction rates using 3DCRT were $38.9 \%$ compared with single PA (range, 29.4-58.5\%) or $47.5 \%$ relative to AP/PA (range, 34.5-67.1\%). The mean esophageal doses were 17.9 \pm 2.3 Gy in single PA, 18.2 \pm 2.2 Gy in AP/PA and 15.3 \pm 1.9 Gy in 3DCRT. The median reduction rate using 3DCRT was $12.8 \%$ compared with single PA or $15.6 \%$ relative to AP/PA. Compared with the single PA or AP/PA 2D plan, 3DCRT reduced the median dose by 13.7 or $1.9 \%$ of the maximum spinal cord dose, respectively, and 14.7 or $2.9 \%$ of the maximum PTV dose, respectively. The

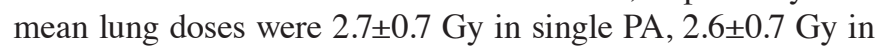
AP/PA and 5.1 \pm 1.0 Gy in 3DCRT. In conclusion, 3DCRT for mid-to-low thoracic spine metastases demonstrated significant dosimetric advantages by reducing the unnecessary irradiation
\end{abstract}

Correspondence to: Dr Seung-Gu Yeo, Department of Radiation Oncology, Soonchunhyang University College of Medicine, Soonchunhyang University Hospital, 31 Soonchunhyang 6-gil, Dongnam-gu, Cheonan 330-721, Republic of Korea

E-mail: md6630@schmc.ac.kr

Key words: spine, metastases, palliation, three-dimensional conformal radiotherapy, dosimetry of critical organs, particularly the heart, and by achieving a homogeneous target dose.

\section{Introduction}

Since improvements in cancer management have increased the life expectancy of patients, bone metastases have become an increasing oncological problem. Patients with bone metastases of the spine present with pain, spinal cord compression with neurological deficits and pathological fractures (1). External beam radiation therapy (RT) is a widely accepted and effective modality for the treatment of spine metastases, achieving symptom palliation in $50-80 \%$ of patients (2).

Conventional two-dimensional (2D) RT techniques for spine metastases utilize a single posteroanterior (PA) field or anteroposterior/posteroanterior (AP/PA) parallel-opposed fields for thoracic-lumbar-sacral spines or parallel-opposed lateral fields for cervical spines (3). These methods are simple and may be readily practiced; however, these methods do not spare adjacent healthy organs from the harmful effects of irradiation. Since the survival of patients with spine metastases continues to improve, a greater number of patients are at risk of radiation toxicity (4). Novel advanced RT technologies, including stereotactic body RT and intensity-modulated RT, provide highly conformal and accurate irradiation, permitting an increased target dose while reducing the unnecessary irradiation of normal structures. However, these technologies are primarily used for selected non-metastatic patients; in addition, the costs of equipment and treatment limit their general use (5).

Three-dimensional conformal RT (3DCRT) is positioned between traditional and recent sophisticated technologies. The number of patients with metastatic cancer who receive 3DCRT has increased gradually; however, the beam placement process remains similar to that of 2DRT (5). Few studies have aimed to improve dose distribution using 3DCRT in palliative RT for spine metastases; therefore, the present study aimed to analyze the dosimetric advantages of 3DCRT plans for mid-to-low thoracic spine (T-spine) metastases in terms of sparing adjacent critical organs.

\section{Materials and methods}

RT planning data of 10 patients with mid-to-low T-spine metastases were used for the present dosimetric analysis. 
The patients were aged between 50 and 81 years old (median, 73 years old); in total, there were six males and four females. Patient characteristics, including the primary tumor type and involved T-spine levels, are presented in Table I. Written informed consent was obtained from all patients.

All patients underwent computed tomography (CT) simulation in the supine position with the arms placed above the head. A 16-slice CT scanner with a 0.3-cm slice thickness was used (Brilliance CT Big Bore; Philips Medical Systems, Cleveland, OH, USA). The clinical target volume included the entire vertebra of the involved index spine plus one vertebra superior and inferior to the index spine. The planning target volume was established by adding a $0.5-\mathrm{cm}$ isotropic set-up margin around the clinical target volume. The superior and inferior borders of the planning target volume (PTV) were limited to inter-vertebral spaces. The critical organs at risk (OARs), including the heart, esophagus, whole lungs and spinal cord, were delineated. The heart was outlined along with the pericardial sac; the superior aspect began at the level of the inferior aspect of the pulmonary artery and extended inferiorly to the apex of the heart. The contour of the esophagus and spinal cord was $5 \mathrm{~cm}$ above and below the extent of the PTV.

All RT plans were created using the Eclipse treatment planning system (Varian Medical Systems, Palo Alto, CA, USA) and 6- or 15-MV photon beams, taking into account inhomogeneity corrections. A 3DCRT plan composed of one PA field plus two posterior oblique (mainly $130^{\circ}$ and $230^{\circ}$ ) wedged fields on each side. The relative contributions of the three fields and the wedge angle were optimized in order to achieve the most homogeneous dose distribution in the PTV. For the purpose of the present study, two 2D plans were retrospectively generated for each patient using previously registered planning CT data. The 2D plans consisted of a single PA field or parallel-opposed AP/PA fields. Unequal weighting (AP:PA, 1:2) was used in the AP/PA plan. The dose normalization point was the center of the PTV in the 3DCRT and AP/PA plans. This point was set at the middle of the vertebral body in the single PA plan based on the International Bone Metastases Consensus Working Party reference points (3). The prescription dose was delivered to the PTV margin, so that $\geq 95 \%$ of the PTV received the prescribed dose. The prescription dose was 30 Gy at 3 Gy per fraction. Representative plans of a typical case are illustrated in Fig. 1. Actual treatments used a 3DCRT plan and were performed using a Novalis Tx system (Varian Medical Systems and BrainLab, Feldkirchen, Germany).

In total, 30 cumulative dose-volume histograms (DVHs) were generated (three different plans for each of the 10 patients). The maximum and mean doses to the heart, esophagus, spinal cord, lung and PTV were calculated. The Wilcoxon signed-rank test was used to analyze the mean differences. Statistical tests were two-sided and performed using SPSS software version 14.0 (SPSS Inc., Chicago, IL, USA). A P-value of $<0.05$ was used to indicate a statistically significant difference between values.

\section{Results}

The comparative DVHs of the three RT plans are shown in Fig. 2. The calculated maximum or mean doses administered

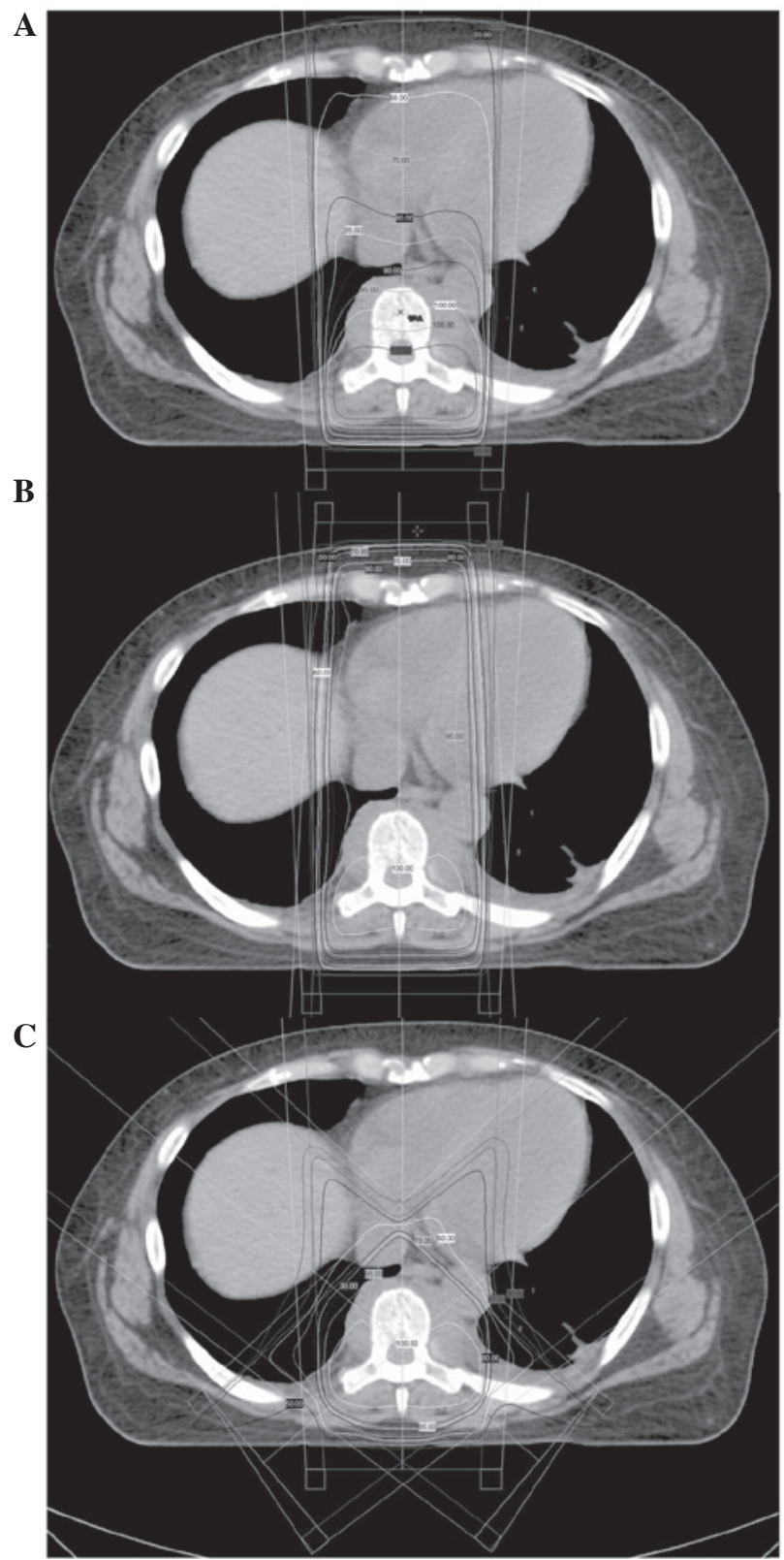

Figure 1. Radiotherapy plans of a typical patient. (A) A single posteroanterior field two-dimensional plan and (B) an opposed anteroposterior/posteroanterior field two-dimensional plan. (C) A three-dimensional conformal plan consisting of a single posteroanterior field and two posterior oblique wedged fields. Isodose lines ( $\geq 30 \%$ of prescription dose) for each plan are also shown.

to the PTV and OARs are listed in Table II. Statistically significant differences were evident for all of the parameters (single PA vs. AP/PA; single PA vs. 3DCRT; AP/PA vs. 3DCRT; $\mathrm{P}<0.05$ ), with the exception of the mean esophageal dose between the single PA vs. AP/PA plan $(\mathrm{P}=0.285)$.

The reduction in the OAR dose using 3DCRT was most prominent for the heart; the mean heart dose was $15.0 \pm 3.1 \mathrm{~Gy}$ in single PA, 17.3 $\pm 4.3 \mathrm{~Gy}$ in AP/PA and $8.5 \pm 1.7 \mathrm{~Gy}$ in 3DCRT. When using 3DCRT, the median percentage reduction rate in the mean heart dose was $38.9 \%$ (range, 29.4-58.5\%) compared with the single PA plan and 47.5\% (range, 34.5-67.1\%) compared with the AP/PA plan. In addition, the median percentage reduction rate in the mean esophageal dose was $12.8 \%$ (range, 4.7-27.9\%) compared with the single PA and 
Table I. Patient characteristics.

\begin{tabular}{cclc}
\hline Patient no. & Age, years/gender & \multicolumn{1}{c}{ Primary tumor } & Involved vertebra \\
\hline 1 & $50 / \mathrm{F}$ & Non-Hodgkin's lymphoma & T7 \\
2 & $59 / \mathrm{F}$ & Uterine cervix cancer & T10 \\
3 & $74 / \mathrm{M}$ & Non-small cell lung cancer & T9 \\
4 & $69 / \mathrm{M}$ & Prostate cancer & T6-7 \\
5 & $74 / \mathrm{M}$ & Non-small cell lung cancer & T8 \\
6 & $54 / \mathrm{M}$ & Thymic cancer & T7 \\
7 & $75 / \mathrm{M}$ & Gall bladder cancer & T9-10 \\
8 & $78 / \mathrm{M}$ & Prostate cancer & T8-10 \\
9 & $81 / \mathrm{F}$ & Breast cancer & T8 \\
10 & $73 / \mathrm{F}$ & Non-small cell lung cancer & T8-9 \\
\hline
\end{tabular}

F, female; M, male; T, thoracic.

Table II. Calculated doses to the PTV and organs at risk according to three radiotherapy plans.

\begin{tabular}{lcccc}
\hline & & \multicolumn{3}{c}{ Mean dose \pm standard deviation (range), Gy } \\
\cline { 3 - 5 } Region & Dose & Single PA & AP/PA & 3DCRT \\
\hline PTV & Maximum & $38.8 \pm 0.5(37.9-39.3)$ & $33.8 \pm 0.8(33.1-35.7)$ & $32.9 \pm 0.6(32.3-34.3)$ \\
Heart & Mean & $15.0 \pm 3.1(11.1-19.7)$ & $17.3 \pm 4.3(12.3-24.5)$ & $8.5 \pm 1.7(6.6-12.1)$ \\
Esophagus & Mean & $17.9 \pm 2.3(15.6-23.0)$ & $18.2 \pm 2.2(15.6-22.5)$ & $15.3 \pm 1.9(12.6-18.6)$ \\
Spinal cord & Maximum & $37.5 \pm 0.6(36.8-38.5)$ & $33.0 \pm 0.6(32.3-34.2)$ & $32.3 \pm 0.4(31.7-32.9)$ \\
Lung & Mean & $2.7 \pm 0.7(1.7-4.0)$ & $2.6 \pm 0.7(1.6-3.9)$ & $5.1 \pm 1.0(3.6-6.8)$ \\
\hline
\end{tabular}

PTV, planning target volume; PA, posteroanterior; AP, anteroposterior; 3DCRT, three-dimensional conformal radiation therapy.

$15.6 \%$ (range, 5.3-29.1\%) compared with the AP/PA plan. The median percentage reduction rate in the maximum spinal cord dose was $13.7 \%$ (range, 12.1-15.6\%) compared with the single PA plan and $1.9 \%$ (range, $0.7-4.0 \%$ ) compared with the AP/PA plan. Furthermore, the median percentage reduction rate in the maximum PTV dose was similar to that of the spinal cord; $14.7 \%$ (range, 12.3-17.6\%) compared with the single PA plan and $2.9 \%$ (range, 1.2-4.0\%) compared with the AP/PA plan.

\section{Discussion}

Palliative RT for spine metastases has long been performed using a 2D technique with an X-ray simulator and portal films; this simple method has advantages in terms of the rapid initiation of treatment for symptom control (5). However, this technique is suboptimal for reducing unnecessary radiation exposure to neighboring healthy tissues (5). The 2D technique is used as these patients have little prospect for long-term survival; therefore, late-manifesting complications do not need to be taken into consideration and the prescribed dose is relatively low when RT is used for palliative reasons (4). However, prolonged survival is possible for certain patients with spine metastases, including those with an oligometastatic status $(6,7)$ or patients with a primary tumor type of favorable histology, such as breast or prostate (1).
The latest and most sophisticated RT technologies, including intensity-modulated RT and stereotactic body RT, are increasingly used by radiation oncology departments $(8,9)$. However, these techniques are primarily indicated for non-metastatic cancers. A survey performed in the United States reported that, in 2007, these technologies were used in $<5 \%$ of metastatic cancer cases (5). 3DCRT is currently classified as intermediate in terms of RT technology advancement. 3DCRT is based upon CT simulation, the visualization of tumors and surrounding anatomy, and an accurate dose-volume calculation (5). To the best of our knowledge, limited data exists concerning the use of 3DCRT for patients with spine metastases and how to optimize beam arrangements and maximize its ability to spare OARs. A study by Soyfer et al (4) reported that the 3DCRT plan for lumbar spine metastases was more effective in terms of bowel and spinal cord exposure compared with the 2D single PA or AP/PA plan. The present study analyzed cases of T-spine metastases and revealed a similar dosimetric advantage of 3DCRT in terms of sparing the heart and esophagus; a reduction in spinal cord exposure was also demonstrated.

Types of radiation-induced cardiac disease include pericarditis, congestive heart failure, cardiomyopathy, valve damage, conduction abnormality, coronary artery disease and myocardial infarction (10). In breast cancer, mortality 
A

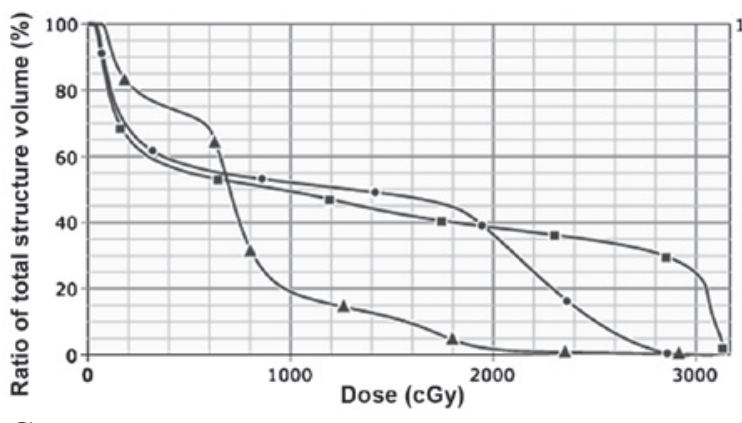

C

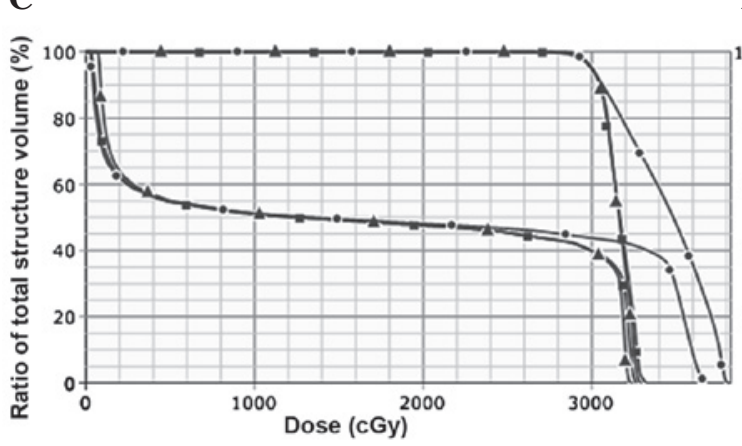

B

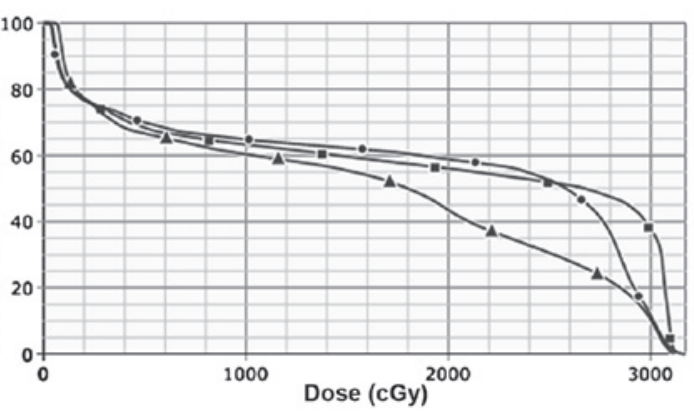

D

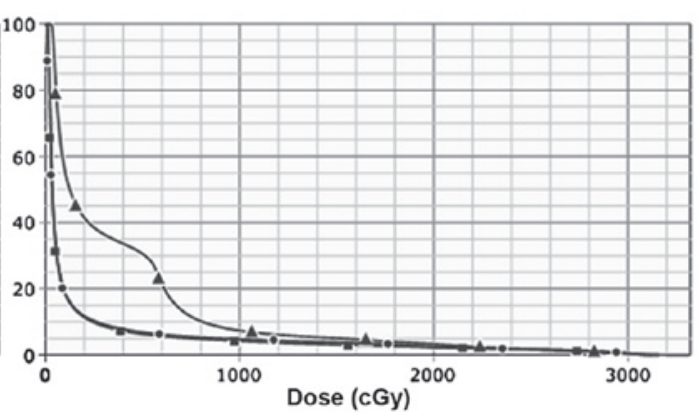

Figure 2. Representative dose-volume histograms of the patient in Fig. 1. (A) Heart, (B) esophagus, (C) planning target volume (upper lines) and spinal cord (lower lines), and (D) lung. Circle, single posteroanterior field plan; box, opposed anteroposterior/posteroanterior fields plan; triangle, three-dimensional conformal plan.

from RT-associated heart disease was reported to offset the improvement of cancer-specific survival due to adjuvant RT $(11,12)$. A clear quantitative dose-volume dependence for the majority of cardiac toxicities remains to be determined (10). A recent study, however, suggested that the rates of major coronary events increase linearly with the mean heart dose by $7.4 \%$ per Gy, with no apparent threshold (13). In addition, it has been established that various clinical parameters may aggravate the risk of radiation-induced heart injury; these include age, diabetes mellitus, smoking, hypertension and the use of cardiotoxic anthracycline-containing chemotherapy $(10,12)$. Re-irradiation for painful spine metastases may be required in patients who achieve no pain relief following initial RT or those who outlive the duration of the first RT response. A recent systematic review indicated that the palliative efficacy of re-irradiation is comparable to that of initial RT (14). The 3DCRT approach used in the present study reduced the mean heart dose by 40-50\% compared with the 2DRT plans. Minimizing the irradiation dose-volume to the heart may therefore assist in the prevention of radiation-induced cardiac toxicities, including mortality. 3DCRT has also been reported to result in a significant dose reduction to the esophagus (15) and spinal cord (16), although the degree of reduction was less than that to the heart.

A 2D plan with a single PA field is frequently used in palliative RT for thoracic and lumbar spine metastases. Regarding the prescription point in this method, opinions in the guidelines from the International Bone Metastases Consensus Working Party were split between the mid-vertebral body and anterior vertebral body, although concerns regarding toxicity were raised for the latter (3). A survey regarding the practice patterns for this RT method concluded that a significantly higher number of respondents used dose prescription to the mid-vertebral body (17). Irrespective of these prescription points, the single PA field is inferior to AP/PA fields in terms of accomplishing homogeneous target dose distribution (18). The International Commission on Radiation Units and Measurements Report 50 recommend a homogeneous dose within 95-107\% of the prescribed dose for the target volume (19). As expected, the present study revealed that the PTV maximum dose was higher with the single PA plan than with the AP/PA plan. In addition, it was demonstrated that 3DCRT could further decrease the PTV maximum dose compared with the AP/PA plan. The PTV maximum dose was also associated with a dose to the spinal cord, as it is located within the target volume.

The mean lung dose was negatively affected by the use of posterior oblique fields in 3DCRT in the present study; however, this increase in lung dose was minimal and appeared insufficient to cause clinical effects (20). 2D plans are performed without CT simulation, and the dose is usually calculated on a single transverse contour taken through the center of the target (21). The actual differences between 2D and 3DCRT plans with regard to dose to the PTV and OARs may be greater than those reported in the present study.

In conclusion, the present study established that, compared with conventional 2DRT plans, 3DCRT for mid-to-low T-spine metastases has dosimetric advantages in terms of reducing unnecessary irradiation to OARs, particularly the heart, and in achieving a homogeneous target dose. Although the radiation doses prescribed for palliative treatments are relatively low, improvements in RT plans are required due to the presence of diverse clinical factors that may lead to aggravation of radiation-induced complications. 


\section{Acknowledgements}

The present study was supported by a grant from the Soonchunhyang University Research Fund.

\section{References}

1. Coleman RE: Clinical features of metastatic bone disease and risk of skeletal morbidity. Clin Cancer Res 12: 6243s-6249s, 2006.

2. Lutz S, Berk L, Chang E, Chow E, Hahn C, Hoskin P, Howell D, Konski A, Kachnic L, Lo S, Sahgal A, et al: Palliative radiotherapy for bone metastases: An ASTRO evidence-based guideline. Int J Radiat Oncol Biol Phys 79: 965-976, 2011.

3. Chow E, Hoskin P, Mitera G, Zeng L, Lutz S, Roos D, Hahn C, van der Linden Y, Hartsell W and Kumar E; International Bone Metastases Consensus Working Party: Update of the international consensus on palliative radiotherapy endpoints for future clinical trials in bone metastases. Int J Radiat Oncol Biol Phys 82: 1730-1737, 2012.

4. Soyfer V, Corn BW, Shtraus N, Schifter D and Tempelhof H: The advantage of 3D conformal treatment of lumbar spine metastases in comparison to traditional PA or AP-PA techniques: Restoring an intermediate niche of therapeutic sophistication. Radiat Oncol 8: 34, 2013.

5. Guadagnolo BA, Huo J, Liao KP, Buchholz TA and Das P: Changing trends in radiation therapy technologies in the last year of life for patients diagnosed with metastatic cancer in the United States. Cancer 119: 1089-1097, 2013.

6. Badakhshi H, Grün A, Stromberger C, Budach V and Boehmer D: Oligometastases: The new paradigm and options for radiotherapy. A critical review. Strahlenther Onkol 189: 357-362, 2013.

7. Yeo SG, Kim DY, Kim TH, Jung KH, Hong YS, Kim SY, Park JW, Choi HS and Oh JH: Curative chemoradiotherapy for isolated retroperitoneal lymph node recurrence of colorectal cancer. Radiother Oncol 97: 307-311, 2010.

8. Kim MJ, Yeo SG, Kim ES, Min CK and Se An P: Intensity-modulated stereotactic body radiotherapy for stage I non-small cell lung cancer. Oncol Lett 5: 840-844, 2013.

9. Kim ES and Yeo SG: Volumetric modulated arc radiotherapy sparing the thyroid gland for early-stage glottic cancer: A dosimetrical analysis. Oncol Lett 7: 1987-1991, 2014.

10. Gagliardi G, Constine LS, Moiseenko V, Correa C, Pierce LJ, Allen AM and Marks LB: Radiation dose-volume effects in the heart. Int J Radiat Oncol Biol Phys 76 (3 Suppl): S77-S85, 2010.
11. Darby SC, McGale P, Taylor CW and Peto R: Long-term mortality from heart disease and lung cancer after radiotherapy for early breast cancer: Prospective cohort study of about 300,000 women in US SEER cancer registries. Lancet Oncol 6: 557-565, 2005.

12. Sung K, Lee KC, Lee SH, Ahn SH, Lee SH and Choi J: Cardiac dose reduction with breathing adapted radiotherapy using self respiration monitoring system for left-sided breast cancer. Radiat Oncol J 32: 84-94, 2014.

13. Darby SC, Ewertz M, McGale P, Bennet AM, Blom-Goldman U, Brønnum D, Correa C, Cutter D, Gagliardi G, Gigante B, Jensen MB, et al: Risk of ischemic heart disease in women after radiotherapy for breast cancer. $\mathrm{N}$ Engl J Med 368: 987-998, 2013.

14. Wong E, Hoskin P, Bedard G, Poon M, Zeng L, Lam H, Vulpe H, Tsao M, Pulenzas N and Chow E: Re-irradiation for painful bone metastases - a systematic review. Radiother Oncol 110: 61-70, 2014.

15. Werner-Wasik M, Yorke E, Deasy J, Nam J and Marks LB: Radiation dose-volume effects in the esophagus. Int J Radiat Oncol Biol Phys 76 (3 Suppl): S86-S93, 2010.

16. Kirkpatrick JP, van der Kogel AJ and Schultheiss TE: Radiation dose-volume effects in the spinal cord. Int J Radiat Oncol Biol Phys 76 (3 Suppl): S42-S49, 2010.

17. Nakamura N, Shikama N, Wada H, Harada H, Nozaki M, Nagakura H, Tago M, Oguchi M and Uchida N: Variability in the point to which single direct field irradiation is prescribed for spinal bone metastases: A survey of practice patterns in Japan. J Radiat Res 54: 1065-1068, 2013.

18. Andic F, Baz Cifci S, Ors Y, Niang U, Dirier A and Adli M: A dosimetric comparison of different treatment plans of palliative spinal bone irradiation: analysis of dose coverage with respect to ICRU 50 Report. J Exp Clin Cancer Res 28: 2, 2009.

19. International Commission on Radiation Units and Measurements (ICRU): ICRU 50: Prescribing, Recording and Reporting Photon Beam Therapy. ICRU Press, Bethesda, MD, 1993.

20. Marks LB, Bentzen SM, Deasy JO, Kong FM, Bradley JD, Vogelius IS, El Naqa I, Hubbs JL, Lebesque JV, Timmerman RD, Martel MK and Jackson A: Radiation dose-volume effects in the lung. Int J Radiat Oncol Biol Phys 76 (3 Suppl): S70-S76, 2010.

21. Parker $\mathrm{W}$ and Patrocinio $\mathrm{H}$ : Clinical treatment planning in external photon beam radiotherapy. In: Radiation Oncology Physics: A Handbook for Teachers and Students. Podgoršak EB (ed). International Atomic Energy Agency, Vienna, pp219-272, 2005 . 